\title{
Opportunities and pitfalls in surface-wave interpretation
}

\author{
Gerard T. Schuster ${ }^{1}$, Jing Li ${ }^{1}$, Kai Lu', Ahmed Metwally ${ }^{1}$, Abdullah AlTheyab ${ }^{1}$, and Sherif Hanafy ${ }^{1}$
}

\begin{abstract}
Many explorationists think of surface waves as the most damaging noise in land seismic data. Thus, much effort is spent in designing geophone arrays and filtering methods that attenuate these noisy events. It is now becoming apparent that surface waves can be a valuable ally in characterizing the near-surface geology. This review aims to find out how the interpreter can exploit some of the many opportunities available in surface waves recorded in land seismic data. For example, the dispersion curves associated with surface waves can be inverted to give the S-wave velocity tomogram, the common-offset gathers can reveal the presence of near-surface faults or velocity anomalies, and back-scattered surface waves can be migrated to detect the location of near-surface faults. However, the main limitation of surface waves is that they are typically sensitive to S-wave velocity variations no deeper than approximately half to one-third the dominant wavelength. For many exploration surveys, this limits the depth of investigation to be no deeper than approximately $0.5-1.0 \mathrm{~km}$.
\end{abstract}

\section{Introduction}

Tucker and Yorston (1973) wrote a book titled Pitfalls in Seismic Interpretation that aimed to reveal how processed seismic reflection sections could lead to misinterpretation of the subsurface geology. They summarize the content of their book in their preface: "The geometry or shape of the reflecting surface is equally tricky. It can turn synclines into anticlines, reverse the throw of faults, superimpose one structure on another by sideswipe, and create a diffraction-anticline. Our latest and perhaps most serious pitfall is computer-derived. The recording and playback can distort both the structure and stratigraphy. Here real structures can be suppressed, false bedding created, faults smeared, and all of the geology lost. Only through constant rapport between the geologist, the interpreter, and the processing engineer will these recording and playback errors be avoided."

In the spirit of Tucker and Yorston (1973), this paper presents a review on interpreting surface waves in recorded data, not as noise but as signal. It will demonstrate how surface waves can be used to estimate the locations of near-surface S-wave velocity anomalies and their extent in depth. Similar to reflection migration, we show how surface waves can be migrated to estimate the locations of near-surface velocity anomalies. The examples in this paper also reveal some pitfalls leading to misinterpretation of surface waves, and their avoidance by using an integrative interpretation of P-wave velocity tomograms, surface-wave migration images, and S-wave velocity tomograms.
This review is organized into four sections. After this brief introduction, we present an intuitive overview of the physics of surface-wave propagation, data processing, and how phase velocities are converted to depth. The next section provides examples of inverting and interpreting surface waves records for several seismic surveys. The examples include surface-wave detection of faults for earthquake hazard analysis, detection of low-velocity anomalies for mineral deposits, and identification of low-velocity zones (LVZs) for statics and near-surface drilling hazards. A summary is presented in the last section.

\section{A quick look at surface waves}

A surface wave mostly propagates along a surface (such as the earth's free surface or the seafloor) and generally attenuates with increasing depth. If the velocity structure of the medium is layered, then different frequency components of the surface waves will propagate laterally with different phase velocities. The horizontal propagation velocity at a specific frequency is a weighted average of the S-wave velocities down to, for Rayleigh waves, half to about a third of the wavelength $(z \approx 1 / 3 \lambda)$ below the free surface (Richart et al., 1970; Park et al., 1999). Thus, the average S-wave velocity to the depth of one-third of the wavelength can be approximated by measuring the corresponding phase velocity associated with that wavelength. Another robust method for estimating depth is to assign different frequencies to

\footnotetext{
${ }^{1}$ King Abdullah University of Science and Technology, Department of Earth Science and Engineering, Thuwal, Saudi Arabia. E-mail: gerard. schuster@kaust.edu.sa; jing.li@kaust.edu.sa; kai.lu@kaust.edu.sa; ahmed.metwally.2@kaust.edu.sa; abdullah.altheyab@kaust.edu.sa; sherif. mahmoud@kaust.edu.sa.

Manuscript received by the Editor 21 January 2016; revised manuscript received 4 November 2016; published online 20 January 2017. This paper appears in Interpretation, Vol. 5, No. 1 (February 2017); p. T131-T141, 15 FIGS.

http://dx.doi.org/10.1190/INT-2016-0011.1. @ 2017 Society of Exploration Geophysicists and American Association of Petroleum Geologists. All rights reserved.
} 
different depths by using the surface-wave sensitivity kernel (Xia et al., 1999; Lin and Ritzwoller, 2010). To compute the actual S-wave velocity model, the surface waves can be inverted using the elastic-wave equation.

There are three types of surface waves that are of keen interest to explorationists: Love waves (see Figure 1a) recorded by horizontal geophones with horizontal-particle motion perpendicular to the propagation direction, Rayleigh waves (see Figure 1b) (Haney and Douma, 2012) recorded by vertical- and horizontal-component geophones, and guided waves (Zhao et al., 1994; Aki and Richards, 2002; Beresford and Janex, 2005; Gaiser and a) Love waves

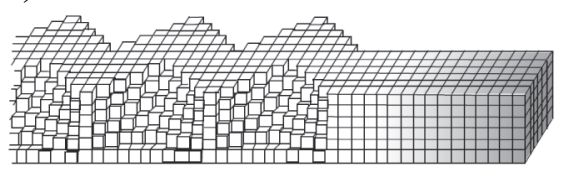

b) Rayleigh waves

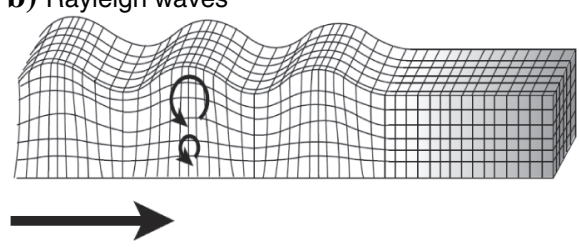

Direction of propagation c) Stoneley waves

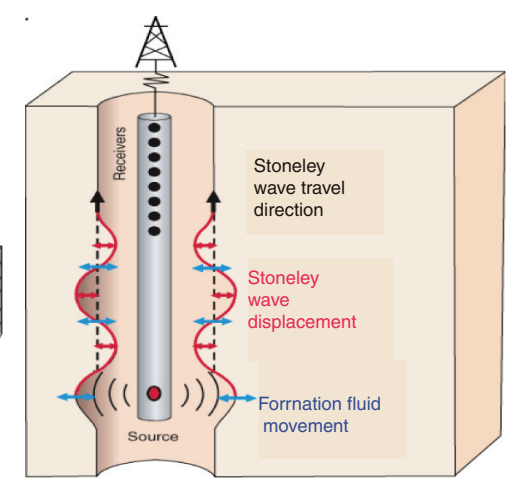

Figure 1. Illustrations of propagating (a) Love waves, (b) Rayleigh waves, and (c) Stoneley waves (USGS, 2016).
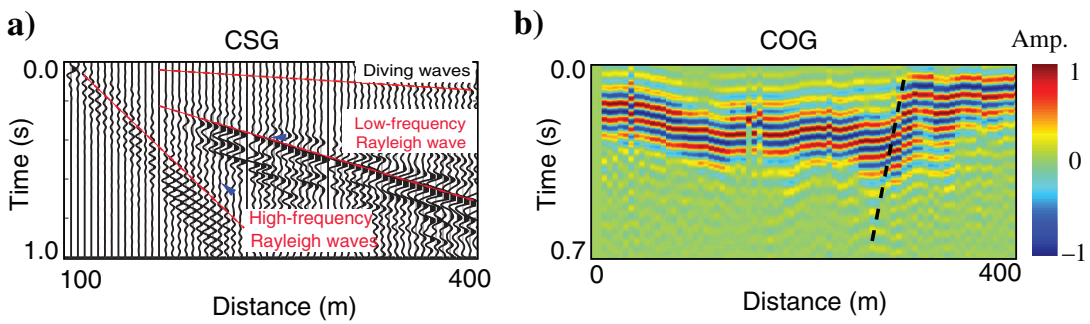

Figure 2. (a) CSG and (b) COG (offset $=50 \mathrm{~m}$ ) recorded by a land survey. The red dashed line in panel (b) is an interpreted normal fault.

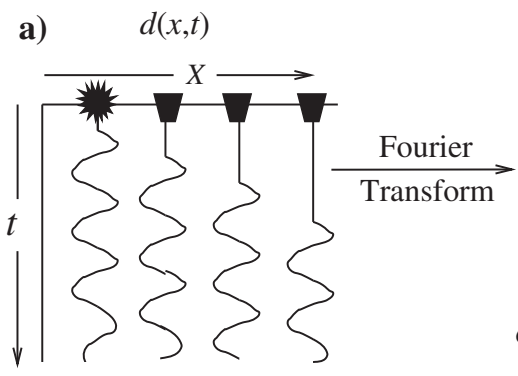

b) Transform of Data

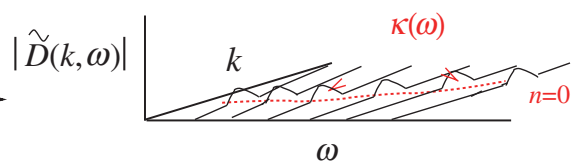

c) Idealized Dispersion Curve

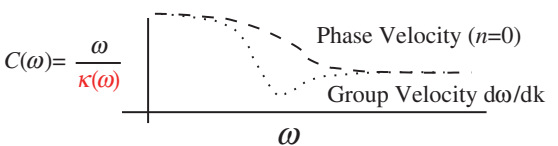

Figure 3. (a) CSG $d(x, t)$, (b) fundamental $(n=0)$ mode dispersion curves, and (c) $C(\omega)-\omega$ curves for Rayleigh waves. Here, the phase velocity is $C(\omega)=\omega / \kappa(\omega)$.
Vasconcelos, 2008; Boiero et al., 2013) that propagate along the water-sediment interface in a borehole (denoted as Stoneley waves in Figure 1c) or the seafloor cholte waves). Amplitudes of Rayleigh and Love waves nerally decrease with depth, so that the motion intal and perpendicular to the direction of propagation. The Stoneley wave is another type of surface wave created along a solid-solid interface or a fluid-solid interface. The Stoneley waves are often exploited in borehole seismic data to infer the S-wave velocities in the formation (Stevens and Day, 1986). Researchers also focused on inverting the dispersion curve for the S-wave velocity profile (Park et al., 1998; Xia et al., 1999; Roy et al., 2013; Li and Hanafy, 2016), imaging lateral heterogeneities using backscattered waves (Hyslop and Stewart, 2015; AlTheyab et al., 2016), and ground-roll analysis (Almuhaidib and Toksöz, 2016; Sloan et al., 2016).

The particle motion for a Rayleigh wave in a layered medium can be retrograde elliptical as denoted by the black ellipse in Figure 1b. For a layered medium, different frequency components of surface waves propagate along the horizontal free surface with different velocities so that the surface waves are denoted as dispersive waves. The result is that an impulse-like source wavelet spreads out in time, i.e., disperses, as it propagates. This is illustrated in Figure 2a, where the high-frequency surface waves propagate at the shallow-layer velocities with a steeper slope in the $x$ - $t$ domain than the low-frequency waves propagating at the faster deeper velocities.

Lateral variations in velocity can be observed in common-offset gathers (COGs), such as the one in Figure $2 b$ that indicates slower velocities on the downthrown side of an interpreted normal fault (dashed black line). If the traces are decomposed into separate frequencies by a 2D FFT, then the fundamental-mode dispersion curve can be picked in the wavenumber frequency $(k-\omega)$ domain (Figure $3 \mathrm{~b})$ or the $C(\omega)-\omega$ domain (Figure 3c). Here, $C(\omega)$ is the phase velocity at frequency $\omega, \tilde{D}(k, \omega)$ is the magnitude spectrum of the traces, and $n=0$ represents the fundamental mode of the Rayleigh waves. Typically, the fundamental mode is characterized by the dispersion curve that follows the largest amount of spectral energy near the $C(\omega)$ axis in Figure 3c. 


\section{Estimation of phase velocity}

Accurately estimating the phase velocity as a function of frequency is the most important first step for accurate inversion of the dispersion curves. There are several methods for estimating the phase velocity, such as the $f-k$ transformation of the shot gathers (Yilmaz, 1987), the phase-shift method (Park et al., 1998), and the slant-stacking algorithm (Xia et al., 2007). We use the following three-step method for estimating the phase velocity as a function of frequency in the $x-t$ domain.

1) Trace normalization is applied to the raw records in Figure $4 \mathrm{a}$ to get the shot gather in Figure $4 \mathrm{~b}$. Window around the surface waves and mute everything else as illustrated in Figure 4c. Apply a narrow band-pass filter $f(t)_{\omega_{o}}$ to the shot gather $d(x, t)_{s}$ centered around $\omega_{o}$ to get $f(t)_{\omega_{o}} \star d(x, t)_{s}=\tilde{d}(x, t)_{s}$ in Figure $4 \tilde{d}$.

2) Apply a linear time shift $\tau(x, \omega)=x / C(\omega)$ to $\tilde{d}(x, t)_{s}$ and stack the events along the offset coordinate:

$$
S\left(t, C\left(\omega_{0}\right)\right)=\sum_{x} \tilde{d}\left(x, t-x / C\left(\omega_{0}\right)\right)_{s} .
$$

An example for the data in Figure 4 is shown in Figure 5 , in which different moveout velocities $C\left(\omega_{0}\right)$ are applied to the same band-passed filter shot gather. The shot gather that is flattened the most is the one that assigns the true phase velocity $C\left(\omega_{0}\right)$ to that moveout velocity.

3) Plotting the phase velocity against the center frequency gives the dispersion curve shown in Figure 3c. Such curves can be inverted (Aki and Richards, 2002) to give the S-wave velocity profile.

An alternative means for computing the phase velocity as a function of frequency is the two-trace method, in which a narrowband filter centered at $\omega_{0}$ is applied to the traces at two adjacent vertical-component geophones. The slope $d x / d t$ is computed from the two filtered traces to estimate the phase velocity at that center frequency $\omega_{0}$. Another method is to use a high-resolution Radon transform applied to the Fourier spectrum of the shot gather (Li and Schuster, 2016a).

\section{Estimating depth versus frequency}

The S-wave velocity model can be used to assess velocity anomalies in the near-surface sediments, which can be used to identify drilling hazards and assign statics corrections (Xia et al., 1999; Socco et al., 2010). Unfortunately, surface-wave characterization of the subsurface with typical exploration surveys fails to reliably generate and record useful signals lower than $2 \mathrm{~Hz}$, which are necessary to sample greater depths. For example, if the lowest frequencies recorded are no less than $2 \mathrm{~Hz}$ and the average S-wave velocity is $1000 \mathrm{~m} / \mathrm{s}$, then surface waves are not sensitive to geology below a depth of half to one-third of the dominant wavelength, or $166 \mathrm{~m}$ in the following case:

$$
z \approx \frac{\lambda_{S}}{3}=\frac{C_{R}}{3 f}=\frac{1}{3} \frac{1000 \mathrm{~m} / \mathrm{s}}{2 \mathrm{~Hz}}=166 \mathrm{~m},
$$

where $f$ is the frequency and $C_{R}$ is the Rayleigh-wave phase velocity at that frequency. The limited depth range can be mitigated by recording ambient noise (Okada and Suto, 2003; Lin et al., 2013) where useful frequencies as low as $0.5 \mathrm{~Hz}$ can be used to probe deeper than $1 \mathrm{~km}$ below the free surface. Acquiring ambient noise seismic data usually requires days or weeks of recording time; however, spatial autocorrelation surveys can sometimes be completed in $20-45 \mathrm{~min}$ of recording time.

The sensitivity kernel is another robust method to estimate the depth of the surface wave. For a layered-earth model, Rayleigh-wave dispersion curves can be calculated by Knopoff's method through a nonlinear and implicit characteristic equation $F\left(f, C_{R}, V_{\mathrm{S}}, V_{\mathrm{P}}, h\right)=0$, where $f$ is the frequency, $C_{R}$ is the phase velocity at frequency $f, V_{\mathrm{S}}$ is the S-wave velocity vector that includes the $\mathrm{S}$-wave velocity of each layer, $V_{\mathrm{P}}$ is the $\mathrm{P}$-wave velocity vector that includes the $\mathrm{P}$-wave velocity of each layer, and $h$ is the layer thickness vector that includes the thickness of each layer (Schwab and Knopoff, 1972). According to the sensitivity analysis of the Rayleighwave phase velocity by Xia et al. (1999), the S-wave velocity is the dominant parameter that influences
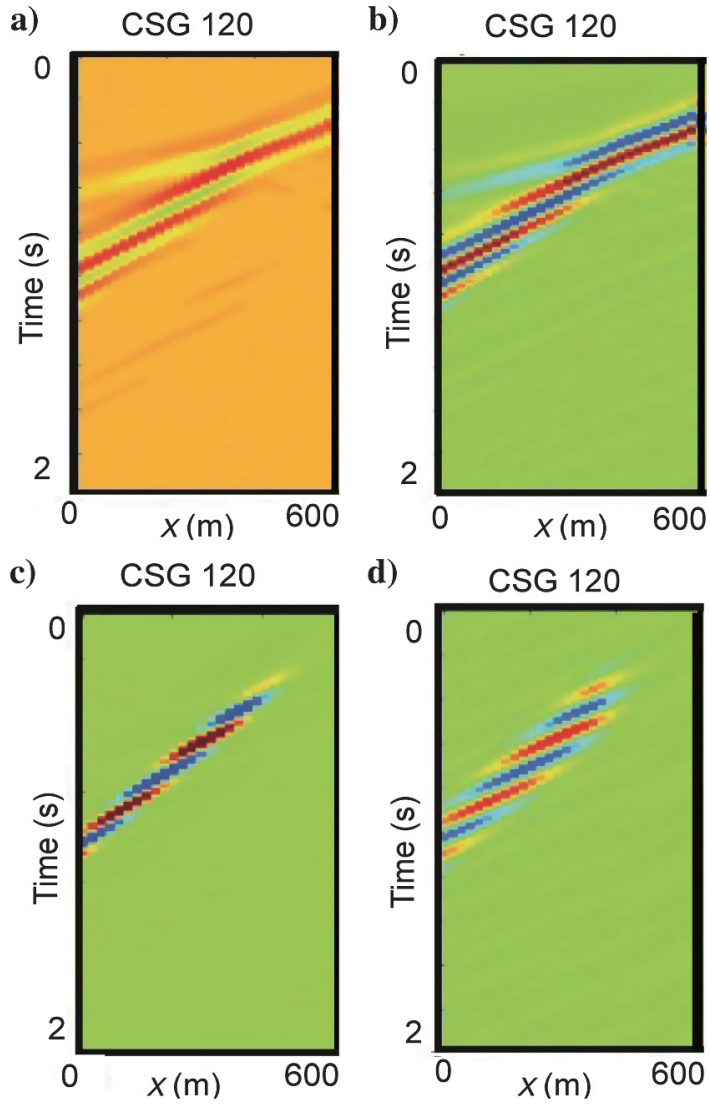

Figure 4. (a) CSG 120 from the synthetic data, (b) CSG after trace normalization, (c) CSG after windowing around the surface waves and muting everything else, and (d) CSG after muting and applying a narrow band-pass filter from 5 to $25 \mathrm{~Hz}$. 
changes in the Rayleigh-wave phase velocity for the fundamental mode. Therefore, ignoring $V_{\mathrm{P}}$ and $h$, the characteristic equation $F$ can be simplified as

$$
F\left(f, V_{\mathrm{S}}, C_{R}\right)=0 .
$$

Then, the phase-velocity sensitivity kernel $\mathbf{J}_{\mathbf{s}}$ can be obtained by

$$
\mathbf{J}_{\mathbf{s}}=-\frac{\partial C_{R}(\boldsymbol{z})}{\partial V_{\mathrm{S}}(\boldsymbol{z})}=-\frac{\partial F / \partial V_{\mathrm{S}}(\boldsymbol{z})}{\partial F / \partial C_{R}(\boldsymbol{z})} \mid \boldsymbol{z}=\boldsymbol{z}_{i}, \quad i=1,2,3, \ldots, n .
$$

Figure 6 shows the workflow for calculating the phase-velocity sensitivity kernel in equation 4 . According to the sensitivity kernel, we can find the most sensitive depth for the surface waves with center frequency $f$.

The S-wave velocity tomogram can be computed by the Park et al. (1998) method for a 1D layer medium or the wave-equation dispersion inversion (WD) method of $\mathrm{Li}$ and Schuster (2016a) for a 2D or 3D medium. Similar to wave-equation traveltime tomography, the complicated surface-wave arrivals in traces are skeletonized as simpler data, namely, the picked dispersion curves in the phase velocity and frequency domains. The a)

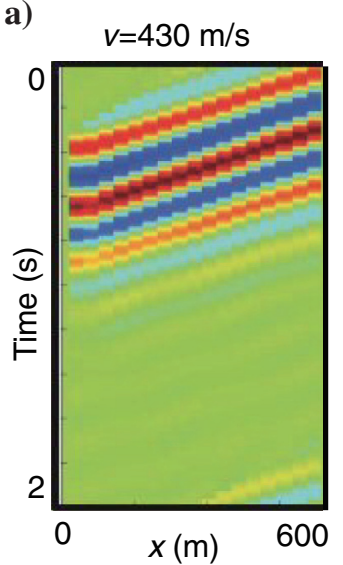

c)

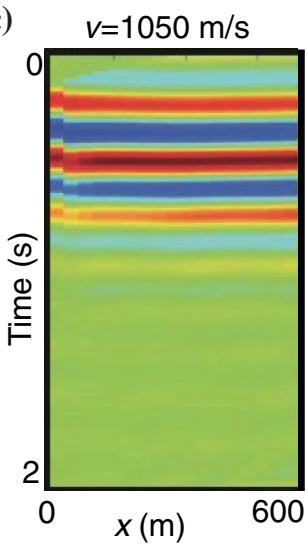

b)

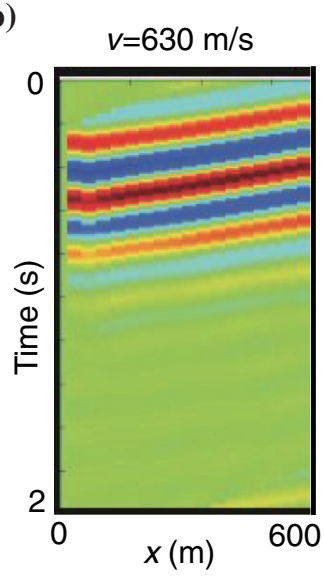

d)

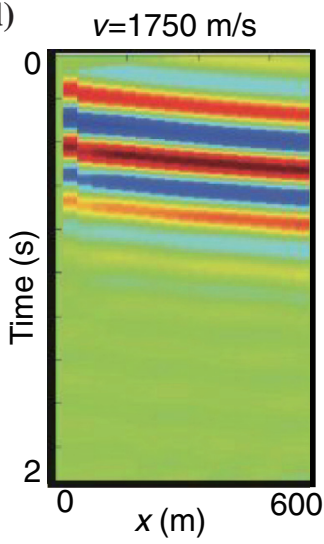

Figure 5. Scans of the filtered traces after applying a linear moveout correction with moveout velocities of (a) 430, (b) 630 , (d) 1050, and (d) $1750 \mathrm{~m} / \mathrm{s}$. Here, the phase velocity is taken to be $1050 \mathrm{~m} / \mathrm{s}$.
WD method computes solutions to the elastic-wave equation and an iterative optimization method is then used to invert these curves for $2 \mathrm{D}$ or 3D S-wave velocity tomograms. The WD procedure not only avoids the assumption of a layered medium, but it also is significantly more robust than full-waveform inversion (FWI) because it replaces complicated surface-wave arrivals with simple dispersion curves in the wavenumber $k_{x}-\omega$ or phase velocity $C(\omega)-\omega$ domains in Figure 3. The limitation of WD is that if the near-surface geology is too complicated, then it is not possible to unambiguously identify and pick coherent dispersion curves.

\section{Interpreting surface-wave records}

With the above background information, let us start interpreting surface waves in the COG domain.

\section{Interpreting faults from COGs}

As Figure 7 illustrates, COGs can reveal fault structures that are almost invisible to standard reflection processing. The reason for this is that surface waves propagate laterally at less than half the velocity of the vertically propagating $\mathrm{P}$-wave reflections so that surface waves have shorter horizontal wavelengths than the P-waves in the same material. These shorter Rayleigh-wave wavelengths lead to much better horizontal resolution than obtained by vertically traveling P-wave reflections. Another advantage of surface waves compared to P-wave reflections is that they can be much more sensitive to lateral velocity variations (Strobbia et al., 2011; Li and Schuster, 2016b).

An example of this sensitivity is shown in Figure 7, in which the COGs in time indicate the locations and shapes of the fault and graben structures. The critical

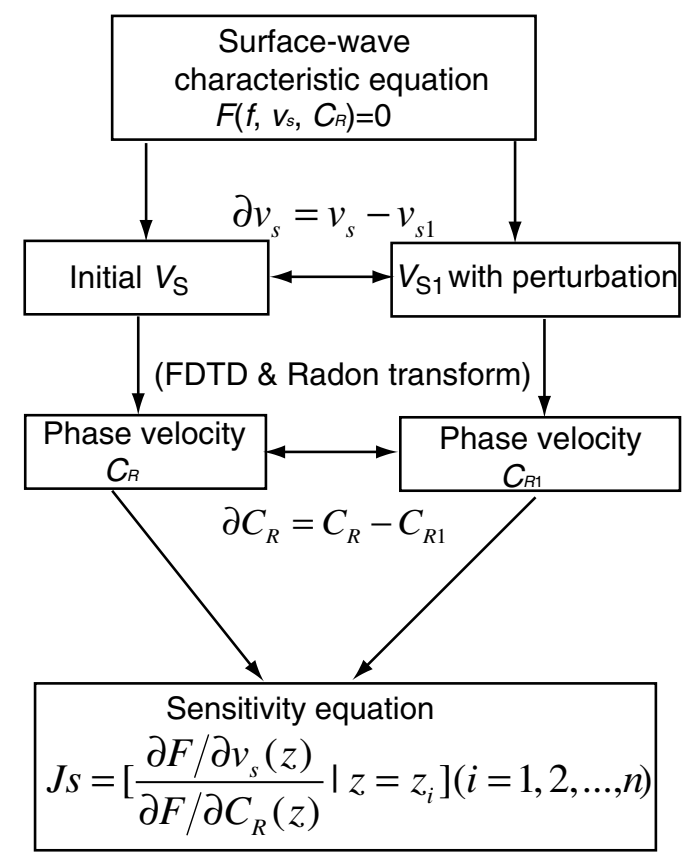

Figure 6. Workflow for calculating the phase-velocity sensitivity kernel. 
source-receiver offset is the one in which the surface waves have fully developed and there is little distortion in the shape (see red solid lines) of the fault model in the COGs. At considerably shorter source-receiver offsets, the surface waves will not have a chance to fully develop, so there will be little indication of the fault in the COGs. As the source-receiver offset becomes wider, the fault structure becomes more distorted. In Figure 7a, the COG events are flat until the midpoint position $x$ of the COGs goes from one side of the fault to the next. It is obvious that the width of the fault $\Delta x^{*}$ can be estimated from the COGs by identifying the value of the source-receiver offset $\Delta x$ where the fault pattern is the narrowest.

The Figure 7 sketches are validated by synthetic simulations of the $2 \mathrm{D}$ elasticwave equation for the fault and graben models. Here, normalized shot gathers were computed for sources and receivers on the free surface, and the commonshot gathers (CSGs) were re-sorted into COGs and are displayed in Figure 8. As the source-receiver offset increases, the delay times of the surface waves increase and the structures appear to be more elongated than the actual model. The depth and actual shape of the graben can be estimated by inverting the dispersion curves, which is later demonstrated in the section with the Qademah data. synthetic model to evaluate the effect of topography on surface-wave dispersion curves. Figure 10a shows the S-wave velocity model with topography, in which 30 vertical-component shot gathers with a shot spacing of $4 \mathrm{~m}$ are computed by the $2 \mathrm{D}$ elastic-wave equation with 60 receivers located every $2 \mathrm{~m}$ on the surface. Figure $10 \mathrm{~b}$ shows the phase-velocity profile with the topography. For a)

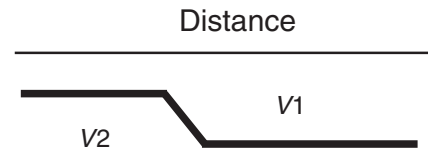

b)

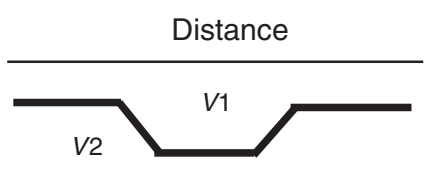

Fault Model

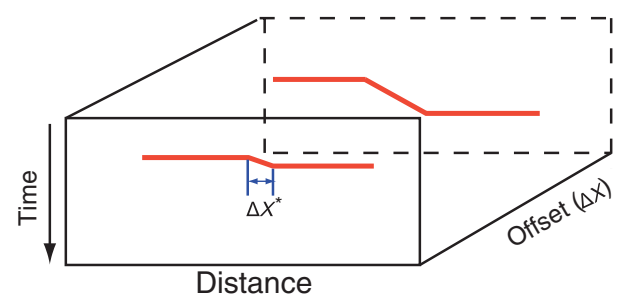

Graben Model

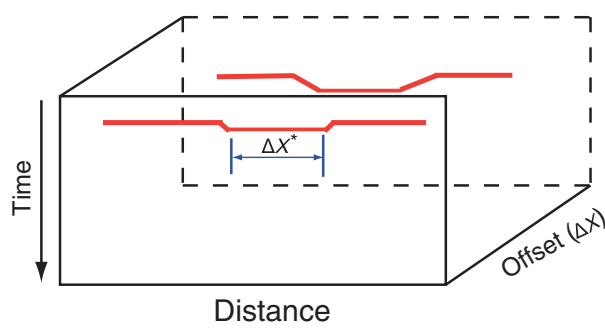

Figure 7. Sketches of (a) fault and (b) graben models. The corresponding COGs, which only contain the first arrival of the surface waves, are plotted against the source-receiver offset. As the source-receiver offset increases, the models estimated from the COGs becomes distorted.

\section{Mining application}

Seismic records are recorded at an open pit mine, and the goal is to use surface waves to identify the nearsurface locations of soft minerals. The seismic source is a $4 \mathrm{~kg}$ sledge hammer, and a shot gather for a source located at each geophone is recorded by 120 geophones spaced at $0.5 \mathrm{~m}$ intervals. The seismic data were recorded along the red line depicted in Figure 9a, and the surface-wave phase velocities as a function of frequency were estimated using the procedure described in Figures 4 and 5 . The resulting phase-velocity image is plotted in Figure 9c, where equation 2 is used to convert from frequency to pseudodepth. According to the geologic survey, the blue LVZs in Figure 9c correspond to the geologic interpretation of rich mineral deposits in Figure $9 \mathrm{~b}$. The blue and red circles in Figure $9 \mathrm{~b}$ show mineral deposits and a dry-soil region, respectively. These rich deposits were interpreted prior to the seismic acquisition and were expected to be characterized by zones of low S-wave velocity.

Figure 9a shows that there is topography that should be accounted for in the processing of the data. A pitfall in the surface-wave method is that the anomalous dispersion associated with buried or topographical obstructions must be accounted for before the inversion procedure is applied. As an example, Figure 10 shows a simple
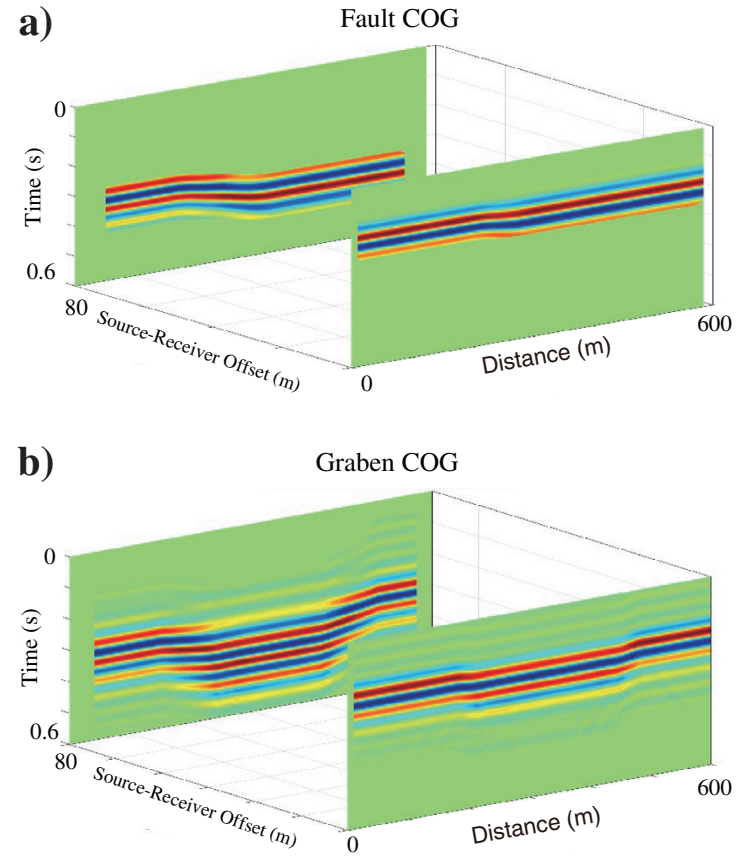

Figure 8. COGs of (a) fault and (b) graben models computed by finite-difference solutions to the $2 \mathrm{D}$ elastic-wave equation. The dominant events in the COGs are those of Rayleigh waves. 
comparison, Figure 10c shows the phase-velocity profile for the same velocity model except that the free surface is flat. Comparing the two results shows that there will be a systematic error in the estimated S-wave velocity tomogram unless the topography is accounted for. For the WD method, topographic effects are automatically accounted for by incorporating the free-surface topography into the finite-difference simulations.

\section{Qademah fault seismic data}

Seismic data were recorded over the Qademah fault located near the Red Sea coast of Saudi Arabia (Hanafy et al., 2015). Surface geologic evidence suggests that this fault extends $25 \mathrm{~km}$ along the north-south direction, and it could be an earthquake risk for the inhabitants of nearby King Abdullah Economic City. Four different survey locations are selected to cross the expected location of the Qademah fault. We applied the early-arrival waveform inversion (EWI) method to near-surface refraction data to obtain a P-wave velocity tomogram (Sheng et al., 2006). Compared with FWI (Tarantola, 1984), EWI avoids a high-frequency assumption but has more reliable convergence properties because it needs to explain only the early arrivals in the recorded traces. The first-arrival traveltimes were picked and inverted (Nemeth et al., 1997) to give the P-velocity tomograms shown in the left column of Figure 11. The right column contains the surface-wave COGs. The LVZs in the P-wave velocity tomograms indicate low-velocity

a)
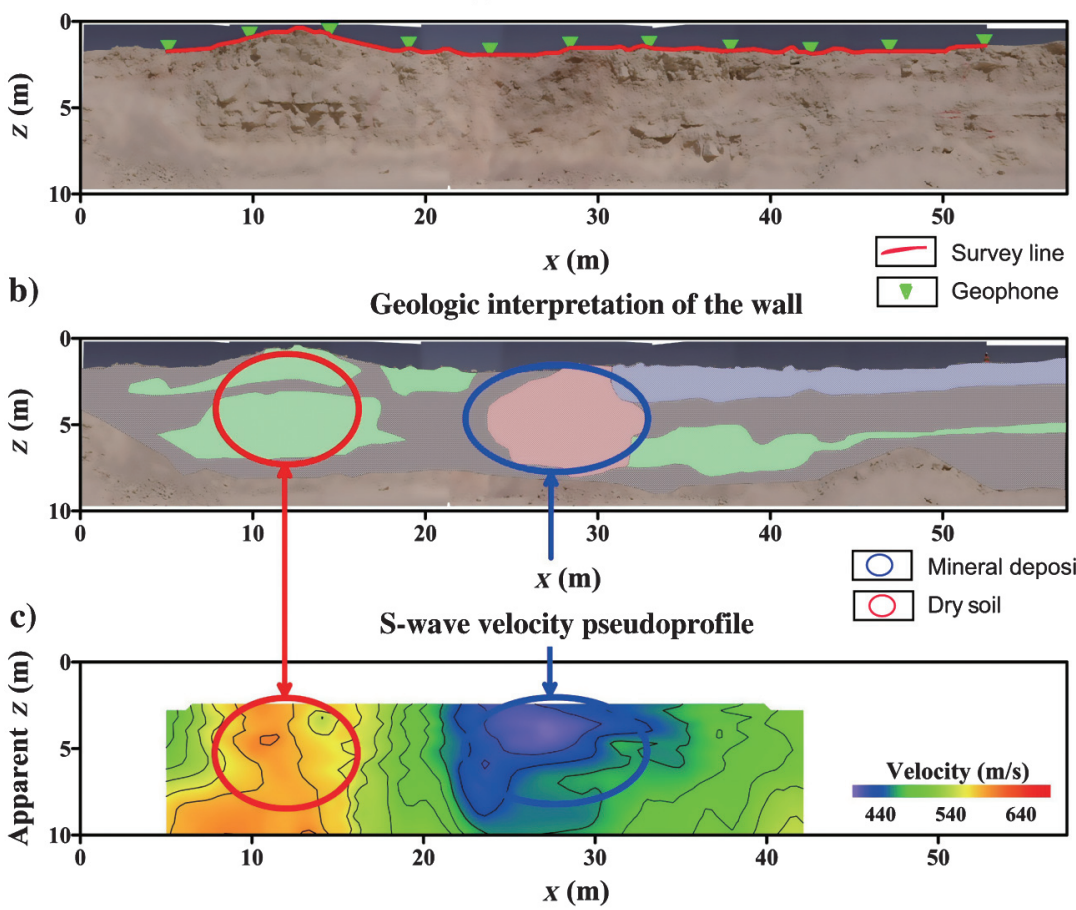

Figure 9. (a) Picture of a rock wall and a seismic line denoted by the red line; the green triangles denote some of the geophone locations, (b) interpreted geology (red circle is dry soil and the blue circle indicates mineral deposits), and (c) S-wave velocity profile computed from the relationship between the depth and wavelength along the seismic survey line. anomalies at the near surface that give rise to the delayed Rayleigh arrivals in the COGs. In addition, there is significant dispersion in the COGs of Figure $11 \mathrm{~b}$ and $11 \mathrm{~d}$ as the Rayleigh waves enter the downthrown side of the normal fault. The solid red lines are interpreted as part of the Qademah fault system, and the yellow arrows in the COGs refer to Rayleigh waves. The COG profile in Figure $11 \mathrm{~h}$ is not consistent with the fault marked as the red dashed line in Figure 11g. The reason is that the depth of this fault is more than $60 \mathrm{~m}$ below the recording surface, which is below the penetration depth of the Rayleigh waves. By combining the interpretation of Rayleigh-wave COGs and P-wave velocity tomograms, faults can be identified with greater certainty (Hanafy, 2015).

\section{East Africa seismic data}

Seismic data are collected over a basin near Olduvai Gorge, Tanzania in East Africa, where the shot spacing is $10 \mathrm{~m}$, the receiver spacing is $5 \mathrm{~m}$, and there are 240 traces per shot gather to give a total of 320 shot gathers. The goals are to estimate the basin depth and the nearsurface geology. Figure 12 depicts a typical shot gather and the associated dispersion curve (Li and Hanafy, 2016). We can easily pick the dispersion curve along the peak amplitude in the phase-velocity image.

According to the WD method, the dispersion curves are inverted and the resulting S-wave velocity tomogram is shown in Figure 13a, in which the dashed lines are interpreted as fault structures. This interpretation is consistent with the interpretation of faults in the Pwave velocity tomogram in Figure $13 \mathrm{~b}$ and the COGs in Figure 13c. Here, a fault is suggested by a sudden delay in the onset of surface waves at the dashed lines. It is to be noticed that the slower S-wave velocity leads to a higher resolution in the S-wave velocity tomogram compared to the P-wave velocity tomogram. The joint interpretation of the S-wave and P-wave velocity tomograms and the COGs strongly suggests the presence of these fault-like structures, which provides a new interpretation to the geology of this basin.

As the Rayleigh wave enters the downthrown side of the normal fault, the arrivals slow down to reveal the graben structures indicated by dashed lines. These COGs are a convenient means for quickly identifying the possible locations of near-surface faults. For example, the dashed white faults are more reliably interpreted than are the dashed red faults because they are indicated by breaks in the COG events as well as localized velocity anomalies in the tomogram. The Swave velocity tomogram inverted from surface waves can sometimes be nonunique so that there are other models that 
can also satisfy the same data. To reduce this uncertainty, higher-order modes or other types of data can also be inverted. Another possibility is $\mathrm{H} / \mathrm{V}$ inversion of microtremor data in which the ratio of the horizontal $\mathrm{H}$ and vertical $\mathrm{V}$ amplitude is inverted to give the depth of the basin. This inversion procedure typically assumes a local $1 \mathrm{D}$ velocity model, knowledge of the $1 \mathrm{D}$ S-wave velocity profile, and the recording of the horizontaland vertical-particle velocities in microtremor data (Nakamura, 1989; Konno and Ohmachi, 1998; BonnefoyClaudet et al., 2006). A more robust approach is to invert for the S-wave velocity tomogram by simultaneous inversion of the dispersion curves and $\mathrm{H} / \mathrm{V}$ data (Lontsi et al., 2016).

\section{Passive seismic data in Long Beach, California}

Ambient noise seismic data were recorded in Long Beach, California, and the primary goal was to assess the area for subsurface oil deposits. A secondary goal was to use the surface waves to identify the subsurface geology for earthquake hazard assessment.

More than 5200 seismometers were deployed (see Figure 14a) to record more than several months of ambient noise data (Lin et al., 2013; AlTheyab et al., 2016). These records were correlated with one another and stacked to compute approximate Green's functions at each receiver. An example of a virtual CSG is shown in
Figure 14b, where it is clear that there is a strong asymmetry between the arrivals at the negative and positive times. This is because the microtremor sources are primarily from ocean waves, and most of the ambient noise is coming from the waves crashing against the shore just to the south of the array.

A 1-Hz narrow-pass filter is applied to the virtual CSGs, and the traveltimes are determined by frequencytime analysis (Bensen et al., 2007), where the smoothed traveltime map for a single shot is shown in Figure 14c. These traveltimes are for fundamental-mode Rayleigh waves that propagate along the surface, and so they can be used to give the phase-velocity map in Figure 14d for Rayleigh waves. The high-order Rayleigh-wave modes are ignored because they are difficult to identify in this data.

Averaging the phase-velocity tomograms for all virtual shot gathers, with a virtual source at each of the geophones, gives the averaged S-wave velocity tomogram in Figure 15c. The colors in this tomogram delineate different geologic regions in the basin that correspond to the illumination depths associated with a 1-Hz surface wave.

In addition, the backscattered surface waves can be migrated (AlTheyab et al., 2016) to give the migration image along the surface (Figure 15a). The sharp features in the migration image roughly correlate with the sharp color transitions in the S-wave velocity tomogram (Figure 15b). This is not surprising because sharp transitions of velocity

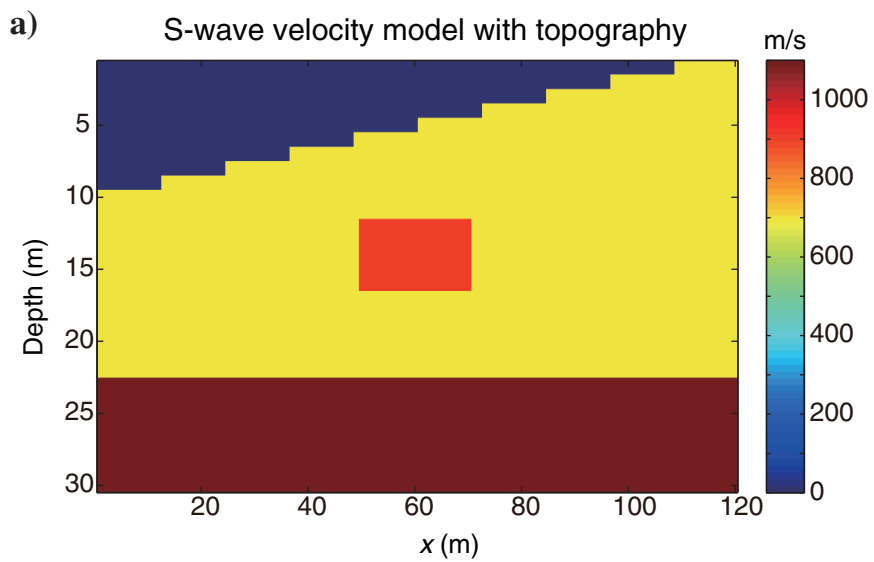

b)

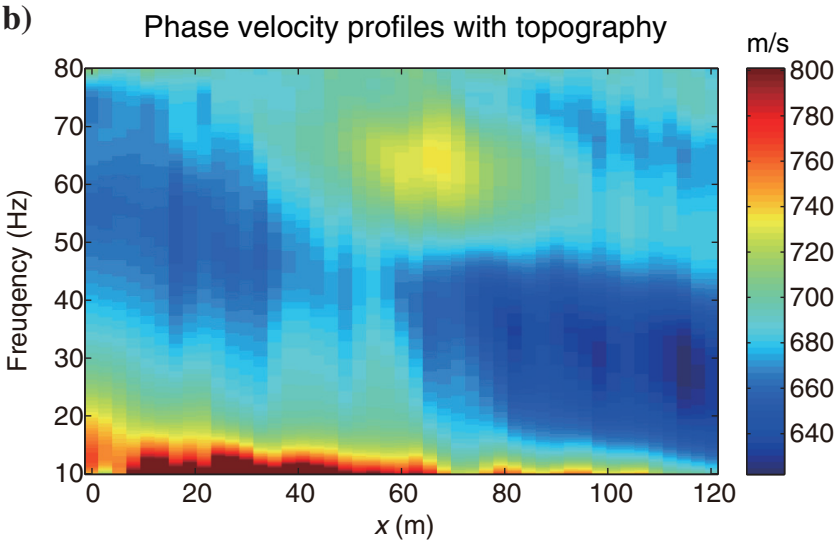

c)

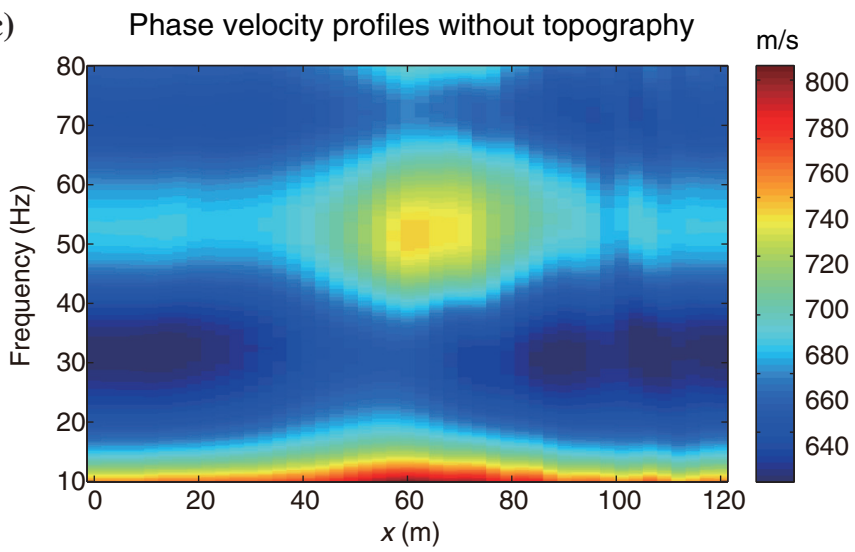

Figure 10. (a) Simple S-wave velocity model with topography, (b) phase-velocity profiles with topography, and (c) phase-velocity profiles without topography. 
can correspond to fault scarps that separate one rock type from another. There is a low-velocity boundary along the white dashed line in the S-wave velocity tomogram, which also coincides with a sharp transition in the migration image. Another prominent feature in both images is a LVZ in the southeast region, which is expected to display relatively low velocities. For exploration geophysics, the combined interpretation of smoothly varying S-wave velocity tomograms and sharply varying migration images can pinpoint areas of strong statics as well as potential hazardous regions for drilling (de Ridder and Dellinger, 2011; de Ridder et al., 2014).

a)

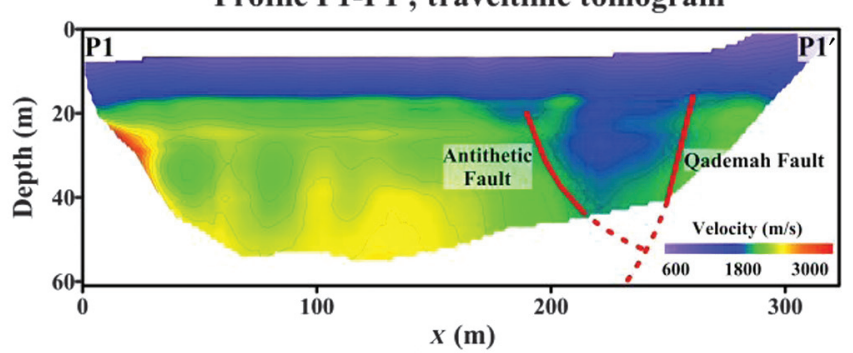

c) Profile P2-P2', traveltime tomogram

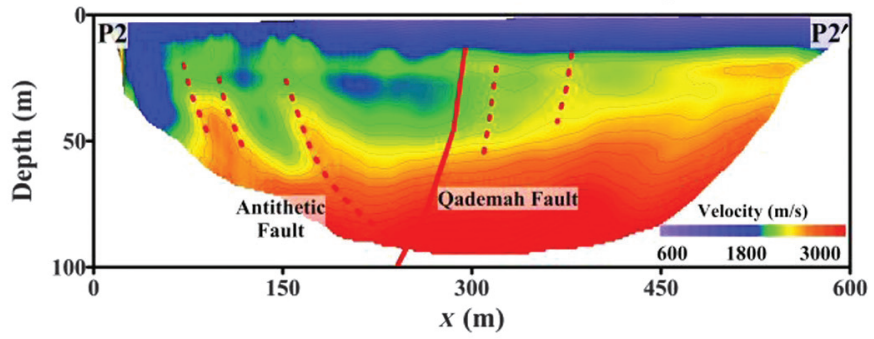

e)

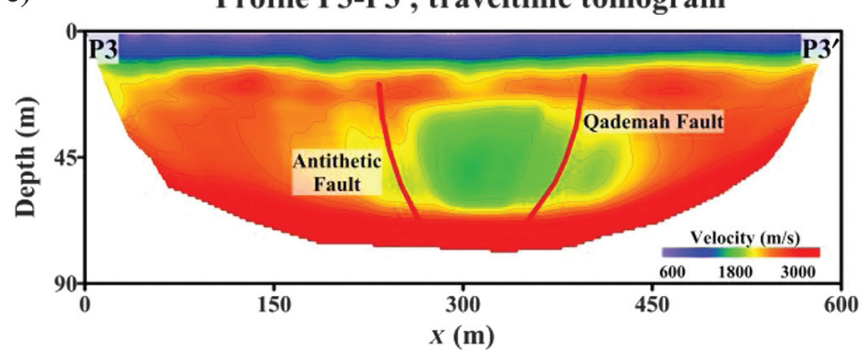

g) Profile P4-P4', traveltime tomogram

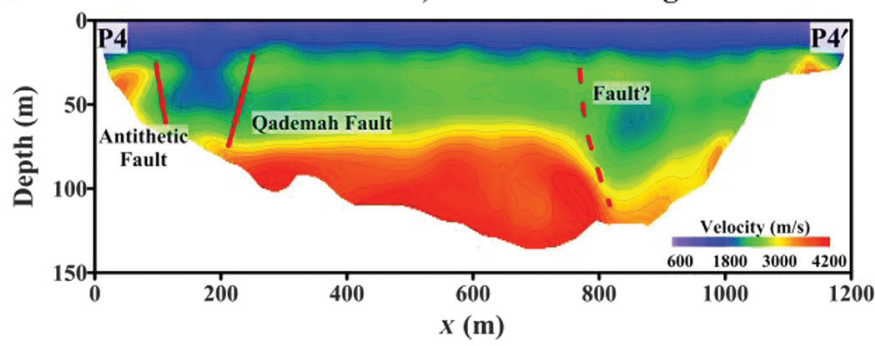

No.20 shot
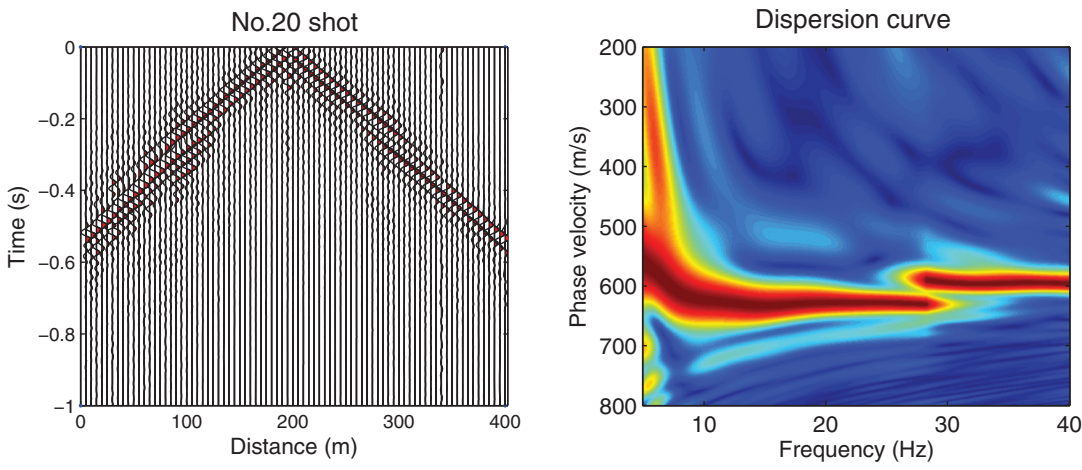

Figure 12. (a) CSG and (b) dispersion curve obtained from an East Africa shot gather. b)

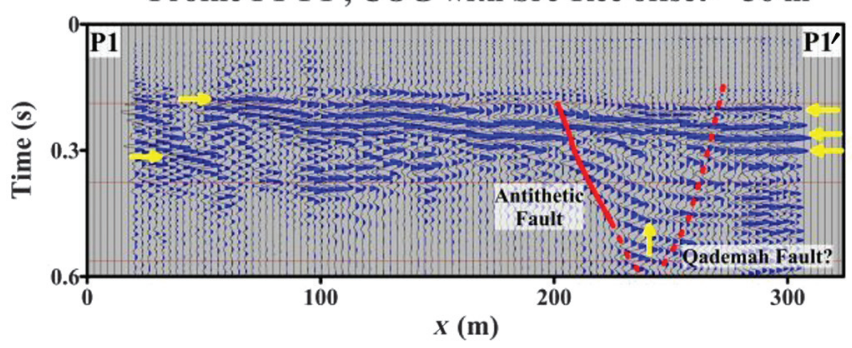

d) Profile P2-P2', COG with Sre-Rec offset $=40 \mathrm{~m}$

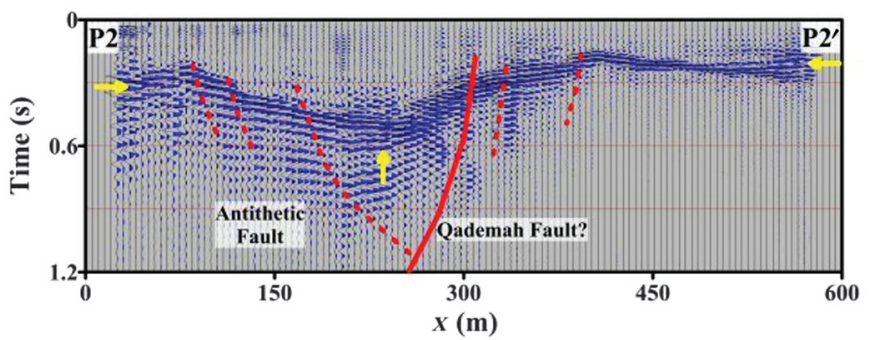

f) Profile P3-P3', COG with Src-Rec offset $=80 \mathrm{~m}$

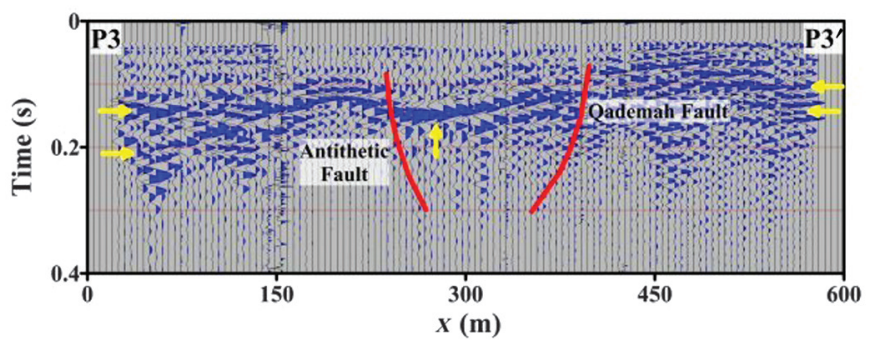

h) Profile P4-P4', COG with Src-Rec offset $=80 \mathrm{~m}$

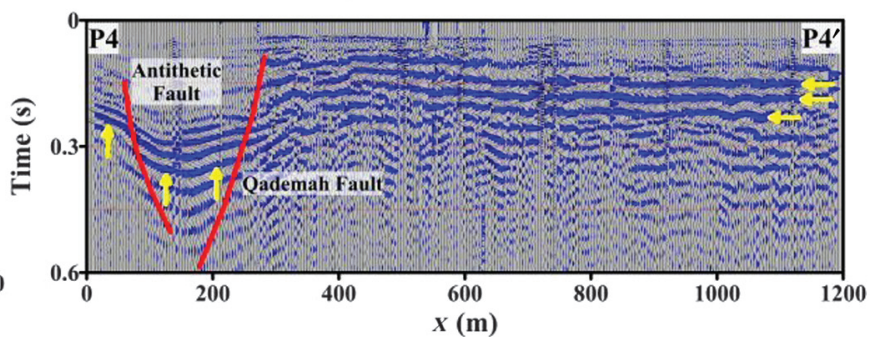

Figure 11. Panels (a, c, e, and g) are P-wave velocity traveltime tomograms associated with four different seismic surveys along the Qademah fault. Panels (b, d, f, and h) are the COGs. 
a)
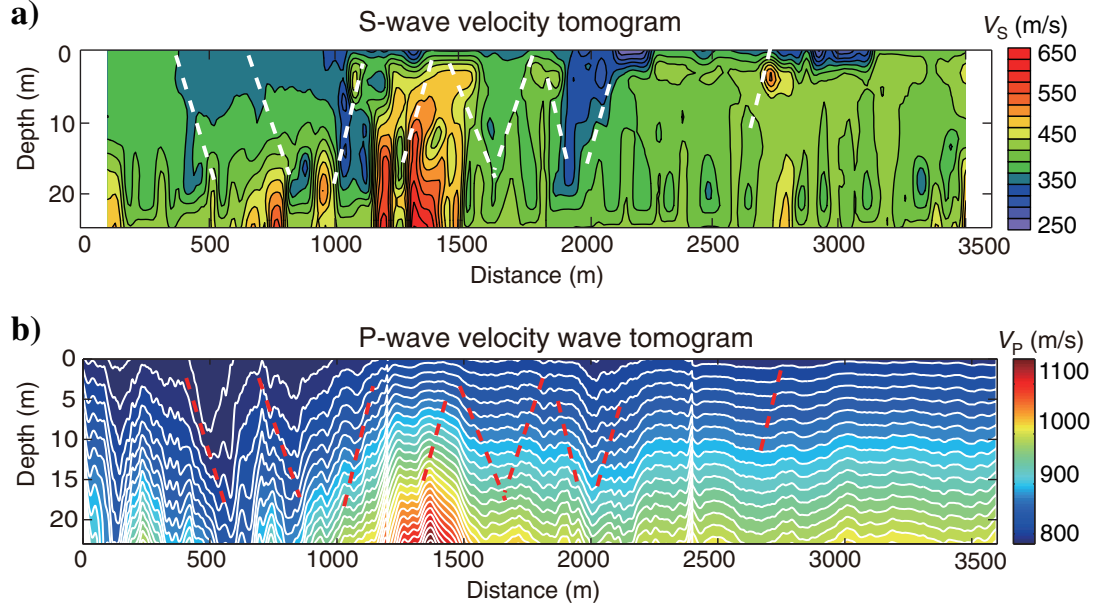

c)

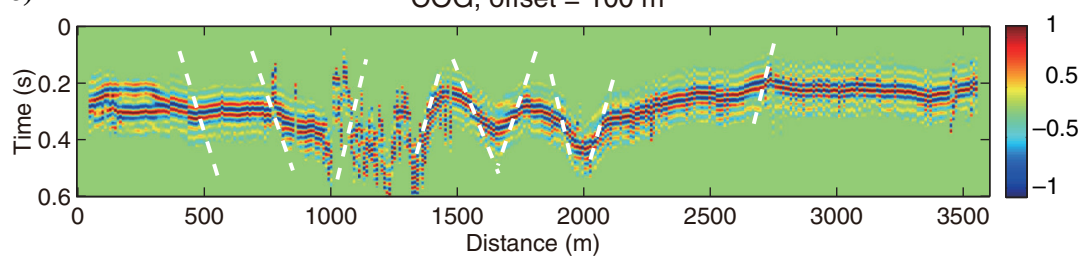

a)

Locations of passive seismometers

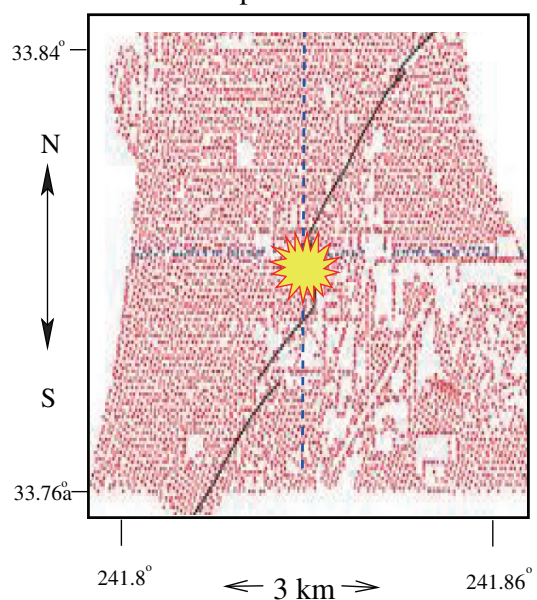

c)

c) Traveltime map: $1 \mathrm{~Hz}$ Rayleigh

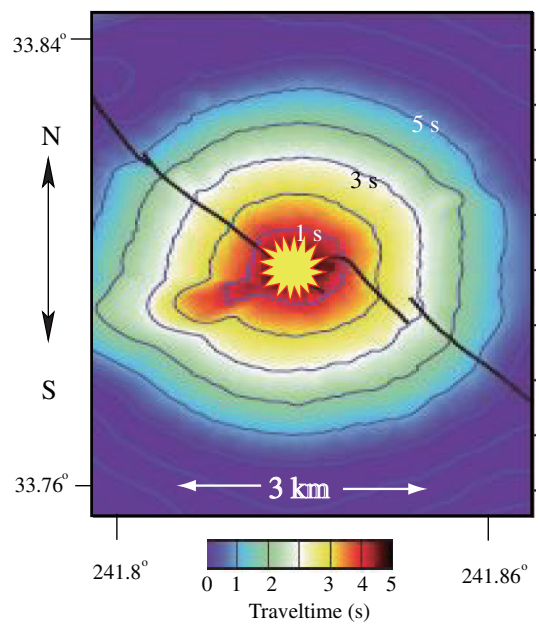

b)

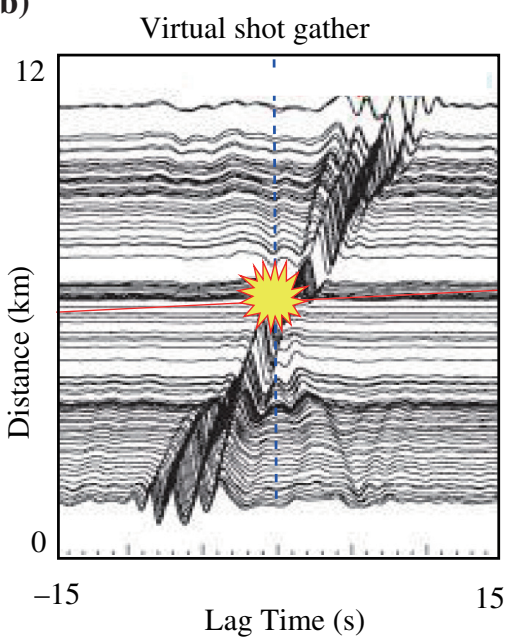

d)

Rayleigh-wave phase velocity

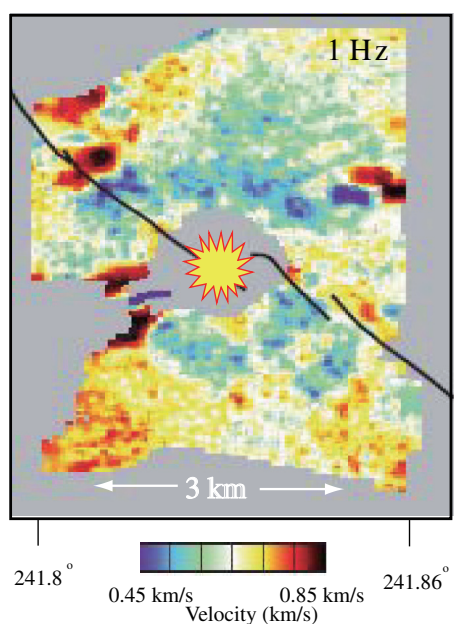

Figure 13. (a) S-wave velocity tomogram obtained by inverting the dispersion curves associated with the East Africa data (see Figure 12), (b) P-wave velocity tomogram by wave-equation traveltime inversion of the first arrivals, and (c) COGs at the source-receiver offset of $100 \mathrm{~m}$ for data recorded by the East Africa survey.
Figure 14. Long Beach passive experiment: (a) The red dots denote locations of passive seismometers, and the blue dashed line is the receiver line for the virtual source denoted by the yellow star, (b) virtual shot gather (with receivers along the blue dashed line in panels [a and b]) computed from ambient noise data recorded for more than two weeks, (c) smoothed traveltimes picked from fundamental Rayleigh-wave arrivals band-limited around the $1 \mathrm{~Hz}$ component of Rayleigh waves, and (d) Rayleigh-wave phase-velocity tomogram reconstructed from one virtual shot gather in the Long Beach passive array data; the color bar at the bottom denotes the Rayleigh-wave velocity values. Images adapted from Lin et al. (2013). 
a)

Surface-wave

migration image

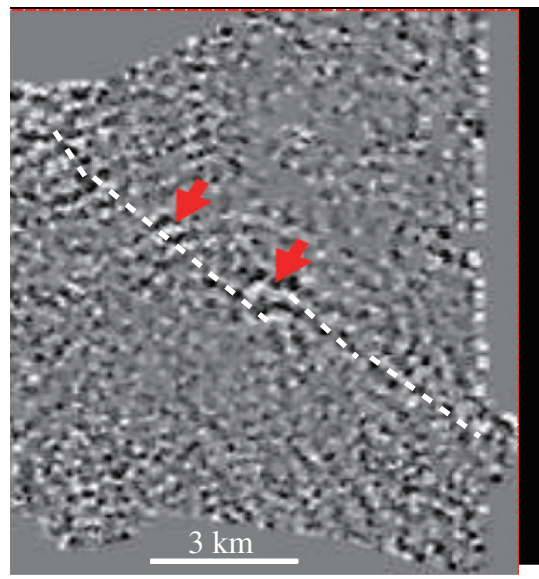

b)

$$
\text { Rayleigh-wave }
$$
velocity tomogram

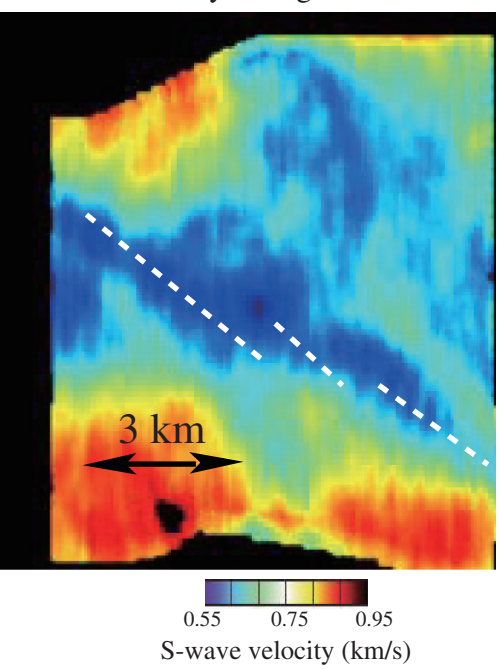

Figure 15. (a) Horizontal slices of the surface-wave migration image (AlTheyab et al., 2016) at the surface and (b) the S-wave velocity tomogram sliced at a depth of $300 \mathrm{~m}$ (Lin et al., 2013) reconstructed from the Long Beach data.

\section{Summary}

We presented a review on how surface waves can be used to estimate the subsurface S-wave velocity distribution. The P-wave and S-wave velocity tomograms can be used in conjunction with COGs of surface waves to identify the location and dip orientation of shallow faults. Lower frequencies lead to deeper penetration of surface waves and the characterization of deeper sediments. The potential pitfall with interpreting faults only from COGs is that rapid delays in the surface-wave arrivals might be indicative of strong lateral contrasts in the S-wave velocity, not necessarily faults. In this case, other methods such as surface-wave migration and Pwave velocity traveltime tomograms should be used to distinguish faults from lateral velocity variations. The main limitation of surface waves is that they are typically sensitive to S-wave velocity variations no deeper than approximately half to one-third of the dominant wavelength. For exploration surveys, this limits the depth of investigation to be no deeper than approximately $0.5-1.0 \mathrm{~km}$. Another potential pitfall is that topographical obstructions and near-surface attenuation can also affect the character of the surface-wave dispersion curves. Without either incorporating or removing these effects, the solution will have a systematic error in the estimated shear velocity.

To summarize, we update in italics a phrase in Tucker and Yorston (1973) so it is applicable to the interpretation of surface-wave images.

"Here, with surface-wave tomograms, real structures can be suppressed, false bedding created, faults smeared, and all of the geology lost. Only through the joint interpretation with other data images, such as migration images, P-wave velocity tomograms and
COGs, and the constant rapport between the geologist, the interpreter, and the processing engineer will misinterpretation be avoided."

\section{Acknowledgments}

We appreciate the financial support from the sponsors of the Consortium of Subsurface Imaging and Fluid Modeling. We also thank KAUST for providing funding by the CRG grant OCRF-2014CRG3-2300 and for the use of the Shaheen computing resources at KAUST.

\section{References}

Aki, K., and P. G. Richards, 2002, Quantitative seismology: University Science Books 1.

Almuhaidib, A. M., and M. N. Toksöz, 2016, Suppression of near-surface scattered body-to-surface waves: A steerable and nonlinear filtering approach: Geophysical Prospecting, 64, 392-405, doi: 10 $.1111 / 1365-2478.12275$.

AlTheyab, A., F. Lin, and G. T. Schuster, 2016, Imaging near-surface heterogeneities by natural migration of backscattered surface waves: Geophysical Journal International, 204, 1332-1341, doi: 10.1093/gji/ ggv511.

Bensen, G., M. Ritzwoller, M. Barmin, A. Levshin, F. Lin, M. Moschetti, N. Shapiro, and Y. Yang, 2007, Processing seismic ambient noise data to obtain reliable broad-band surface wave dispersion measurements: Geophysical Journal International, 169, 1239-1260, doi: 10.1111/j .1365-246X.2007.03374.x.

Beresford, G., and G. Janex, 2005, A practical approach to OBC summation and geophone calibration in areas of shallow water and hard seafloor: 9th International Congress of the Brazilian Geophysical Society, 1466-1470.

Boiero, D., E. Wiarda, and P. Vermeer, 2013, Surface-and guided-wave inversion for near-surface modeling in land and shallow marine seismic data: The Leading Edge, 32, 638-646, doi: 10.1190/tle32060638.1.

Bonnefoy-Claudet, S., F. Cotton, and P.-Y. Bard, 2006, The nature of noise wavefield and its applications for site effects studies: A literature review: Earth-Science Reviews, 79, 205-227, doi: 10.1016/j.earscirev.2006.07.004.

de Ridder, S., B. Biondi, and R. Clapp, 2014, Time-lapse seismic noise correlation tomography at Valhall: Geophysical Research Letters, 41, 6116-6122, doi: 10 .1002/2014GL061156.

de Ridder, S., and J. Dellinger, 2011, Ambient seismic noise eikonal tomography for near-surface imaging at Valhall: The Leading Edge, 30, 506-512, doi: 10.1190/1.3589108.

Gaiser, J., and I. Vasconcelos, 2008, Elastic interferometry for OBC data - Theory and examples: 78th Annual International Meeting, SEG, Extended Abstracts, 1073-1077. 
Hanafy, S. M., 2015, Mapping the Qademah fault with traveltime, surface-wave, and resistivity tomograms: 85th Annual International Meeting, SEG, Expanded Abstracts, 3347-3351.

Hanafy, S. M., A. AlTheyab, and G. T. Schuster, 2015, Controlled noise seismology: 85th Annual International Meeting, SEG, Expanded Abstracts, 5102-5106.

Haney, M. M., and H. Douma, 2012, Rayleigh-wave tomography at Coronation field, Canada: The topography effect: The Leading Edge, 31, 54-61, doi: 10.1190/1 .3679328

Hyslop, C., and R. R. Stewart, 2015, Imaging lateral heterogeneity using reflected surface waves: Geophysics, 80, no. 3, EN69-EN82, doi: 10.1190/geo2014-0066.1.

Konno, K., and T. Ohmachi, 1998, Ground-motion characteristics estimated from spectral ratio between horizontal and vertical components of microtremor: Bulletin of the Seismological Society of America, 88, 228-241.

Li, J., and S. Hanafy, 2016, Skeletonized inversion of surface wave: Active source versus controlled noise comparison: Interpretation, 4, no. 3, SH11-SH19, doi: 10.1190/INT-2015-0174.1.

Li, J., and G. Schuster, 2016a, Skeletonized wave equation of surface wave dispersion inversion: 86th Annual International Meeting, SEG, Expanded Abstracts, 3630-3635.

Li, J., and G. T. Schuster, 2016b, Ray map surface wave migration: Interpretation, 4, no. 4, SQ33-SQ40, doi: 10.1190/INT-2016-0014.1.

Lin, F., D. Li, R. W. Clayton, and D. Hollis, 2013, Highresolution $3 \mathrm{D}$ shallow crustal structure in Long Beach, California: Application of ambient noise tomography on a dense seismic array: Geophysics, 78, no. 4, Q45-Q56, doi: 10.1190/geo2012-0453.1.

Lin, F., and M. H. Ritzwoller, 2010, Empirically determined finite frequency sensitivity kernels for surface waves: Geophysical Journal International, 182, 923-932, doi: 10.1111/(ISSN)1365-246X.

Lontsi, A., M. Ohrnberger, and F. Krüger, 2016, Shear wave velocity profile estimation by integrated analysis of active and passive seismic data from small aperture arrays: Journal of Applied Geophysics, 130, 37-52, doi: 10 .1016/j.jappgeo.2016.03.034.

Nakamura, Y., 1989, A method for dynamic characteristics estimation of subsurface using microtremor on the ground surface: Railway Technical Research Institute, Quarterly Reports 30, 25-33.

Nemeth, T., E. Normark, and F. Qin, 1997, Dynamic smoothing in crosswell traveltime tomography: Geophysics, 62, 168-176, doi: 10.1190/1.1444115.

Okada, H., and K. Suto, 2003, The microtremor survey method: SEG with the cooperation of SEG of Japan and ASEG.

Park, C. B., R. D. Miller, and J. Xia, 1998, Imaging dispersion curves of surface waves on multi-channel record: 68th Annual International Meeting, SEG, Expanded Abstracts, 1377-1380.
Park, C. B., R. D. Miller, and J. Xia, 1999, Multichannel analysis of surface waves: Geophysics, 64, 800-808, doi: 10.1190/1.1444590.

Richart, F. E., J. R. Hall, and R. D. Woods, 1970, Vibrations of soils and foundations: Prentice-Hall.

Roy, N., R. SankarJakka, and H. Wason, 2013, Effect of surface wave inversion non-uniqueness on $1 \mathrm{D}$ seismic ground response analysis: Natural Hazards, 68, 11411153, doi: 10.1007/s11069-013-0677-z.

Schwab, F., and L. Knopoff, 1972, Fast surface wave and free mode computations: Methods in Computational Physics, 11, 87-180.

Sheng, J., A. Leeds, M. Buddensiek, and G. T. Schuster, 2006, Early arrival waveform tomography on near-surface refraction data: Geophysics, 71, no. 4, U47-U57, doi: 10.1190/1.2210969.

Sloan, S. D., J. Tyler Schwenk, and R. H. Stevens, 2016, An example of extreme near-surface variability in shallow seismic reflection data: Interpretation, 4, no. 3, SH1-SH9, doi: 10.1190/INT-2015-0215.1.

Socco, L. V., S. Foti, and D. Boiero, 2010, Surface-wave analysis for building near-surface velocity models established approaches and new perspectives: Geophysics, 75, no. 5, 75A83-75A102, doi: 10.1190/1.3479491.

Stevens, J. L., and S. M. Day, 1986, Shear velocity logging in slow formations using the Stoneley wave: Geophysics, 51, 137-147, doi: 10.1190/1.1442027.

Strobbia, C., A. Laake, P. Vermeer, and A. Glushchenko, 2011, Surface waves: Use them then lose them Surface-wave analysis, inversion and attenuation in land reflection seismic surveying: Near Surface Geophysics, 9, 503-514, doi: 10.3997/1873-0604.2011022.

Tarantola, A., 1984, Inversion of seismic reflection data in the acoustic approximation: Geophysics, 49, 1259-1266, doi: 10.1190/1.1441754.

Tucker, P. M., and H. J. Yorston, 1973, Pitfalls in seismic interpretation: SEG.

USGS, 2016, Earthquake glossary, http://earthquake.usgs. gov/learn/glossary/?term=Rayleigh\%20wave, accessed 10 January 2017.

Xia, J., R. D. Miller, and C. B. Park, 1999, Estimation of nearsurface shear-wave velocity by inversion of Rayleigh waves: Geophysics, 64, 691-700, doi: 10.1190/1.1444578.

Xia, J., Y. Xu, and R. D. Miller, 2007, Generating an image of dispersive energy by frequency decomposition and slant stacking: Pure and Applied Geophysics, 164, 941-956, doi: 10.1007/s00024-007-0204-9.

Yilmaz, O., 1987, Seismic signal processing: SEG.

Zhao, X., M. N. Toksoz, and C. H. Cheng, 1994, Stoneley wave propagation across borehole permeability heterogeneities: Technical Report, Massachusetts Institute of Technology, Earth Resources Laboratory.

Biographies and photographs of the authors are not available. 